\title{
ENERGY FLUX FOR PLANE WAVES IN LINEAR CONSERVATIVE SYSTEMS
}

\author{
M. HAYES \\ (Received 18 August 1976)
}

\begin{abstract}
The point form of the conservation of energy equation is used to give simple and direct proof of results concerning the mean energy flux vector for systems of sinusoidal small amplitude waves in linear conservative systems. No constitutive equation is used explicitly.
\end{abstract}

\section{Introduction}

This note deals with the propagation of infinitesimal plane sinusoidal waves in linear conservative systems. A key element in the study of such waves is the mean energy flux vector, where the mean is taken over a cycle. The basic idea here is that some results concerning the mean energy flux vector for such systems may be deduced directly from the point form of the conservation of energy equation. Apart from one specific example in Section 4, no constitutive equation is used explicitly. The system may or may not be subject to internal constraints such, for example, as the constraint of incompressibility in the case of elastic bodies.

What follows rests upon the following three assumptions.

(i) The energy flux vector is a product of two field quantities.

For example, in the case of a mechanical system in which the Cauchy stress is denoted by $t_{i j}$ and the particle velocity by $v_{i}$ the energy flux vector is $R_{i}$ given [4] by

$$
R_{i}=-t_{j i} v_{j}
$$

Similarly, in an electromagnetic system in which the electric field is denoted by $\mathbf{E}$ and the magnetic field is denoted by $\mathbf{H}$, the energy flux (Poynting) vector is [2]

$$
\mathbf{R}=\mathbf{E} \wedge \mathbf{H} \text {. }
$$

(ii) There is neither internal energy supply nor dissipation.

† The summation convention is used throughout. Repeated Latin subscripts are summed over $1,2,3$. 
If the internal energy is denoted by $\mathscr{E}$ then the point form of the conservation of energy equation is

$$
\frac{\partial \mathscr{E}}{\partial t}+\frac{\partial R_{i}}{\partial x_{i}}=0
$$

(iii) The systems are linear in the sense that if one field quantity such as displacement, velocity, stress, electric field or magnetic field, for example, is of the form

$$
A \exp i \omega(\mathbf{S} \cdot \mathrm{x}-t)
$$

where $\omega$ is real, $\mathbf{S}$ is a complex vector and $\mathbf{A}$ may be a scalar, vector or tensor, then every other field quantity is of the similar form:

$$
\text { B expi } \omega(\mathbf{S} . \mathbf{x}-t) \text {, }
$$

where B may be a scalar, vector or tensor. The form of the constitutive equation is implicit in this assumption.

It may be noted that since the energy flux vector arises as a product of two field quantities it follows that the energy flux vector for two fields is not equal, in general, to the vector sum of the energy flux vectors for the individual fields.

\section{Plane surface waves}

Consider first an infinite train of sinusoidal small amplitude waves propagating along a plane surface $\mathbf{m} \cdot \mathbf{x}=0$. The train may be represented by

$$
\mathbf{A} \exp i \omega\left(\mathbf{S}^{(1)} \cdot \mathbf{x}-t\right) \exp -\alpha_{1} \mathbf{m} \cdot \mathbf{x},
$$

where $\mathbf{A}$ is a complex scalar, vector or tensor, and $\omega, \mathbf{S}^{(1)}$ and $\alpha_{1}$, are real. In linear conservative systems, for such a train, the mean energy flux vector is parallel to the surface ([1]; [5] for the elastic case).

Suppose now that there are two such trains. Let the second be represented by

$$
B \exp i \omega\left(\mathbf{S}^{(2)} \cdot \mathbf{x}-t\right) \exp -\alpha_{2} \mathbf{m} \cdot \mathbf{x},
$$

where $\mathbf{B}$ is a complex scalar, vector or tensor, and $\mathbf{S}^{(2)}, \alpha_{2}$ are real.

Now, of course, some boundary condition or conditions will have to be satisfied on the surface $\mathbf{m} \cdot \mathbf{x}=0$. In the case of an elastic body the boundary conditions could, for example, be that the surface be free, or held fixed, or that the surface be stroked. Whatever the conditions, whether or not they involve products or sums of two or more field quantities, it follows from the assumed linearity of the system that the wave trains must have the same frequency and that the slownesses must satisfy

$$
\mathbf{S}^{(1)}-\mathbf{S}^{(2)}=\left(\mathbf{S}^{(1)} \cdot \mathbf{m}-\mathbf{S}^{(2)} \cdot \mathbf{m}\right) \mathbf{m}
$$


The form of the energy flux vector for the combined motion is $\dagger$

$$
\begin{aligned}
\mathbf{R}= & {\left[\lambda+\cos 2 \omega\left(\mathbf{S}^{(1)} \cdot \mathbf{x}-t\right)-\lambda-\sin 2 \omega\left(\mathbf{S}^{(1)} \cdot \mathbf{x}-t\right)\right] \exp -2 \omega \alpha_{1} \mathbf{m} \cdot \mathbf{x} } \\
& +\left[\boldsymbol{\mu}^{+} \cos 2 \omega\left(\mathbf{S}^{(2)} \cdot \mathbf{x}-t\right)-\mu^{-} \sin 2 \omega\left(\mathbf{S}^{(2)} \cdot \mathbf{x}-t\right)\right] \exp -2 \omega \alpha_{2} \mathbf{m} \cdot \mathbf{x} \\
& +\boldsymbol{\theta}^{(1)} \exp -2 \omega \alpha_{1} \mathbf{m} \cdot \mathbf{x}+\boldsymbol{\theta}^{(2)} \exp -2 \omega \alpha_{2} \mathbf{m} \cdot \mathbf{x} \\
& +\left\{\boldsymbol{\phi}^{+} \cos \omega\left[\left(\mathbf{S}^{(1)}+\mathbf{S}^{(2)}\right) \cdot \mathbf{x}-2 t\right]\right. \\
& \left.-\boldsymbol{\phi}^{-} \sin \omega\left[\left(\mathbf{S}^{(1)}+\mathbf{S}^{(2)}\right) \cdot \mathbf{x}-2 t\right]\right\} \exp -\omega\left(\alpha_{1}+\alpha_{2}\right) \mathbf{m} \cdot \mathbf{x} \\
& +\left[\Psi^{+} \cos \omega\left(\mathbf{S}^{(1)}-\mathbf{S}^{(2)}\right) \cdot \mathbf{x}\right. \\
& \left.-\Psi^{-} \sin \omega\left(\mathbf{S}^{(1)}-\mathbf{S}^{(2)}\right) \cdot \mathbf{x}\right] \exp -\omega\left(\alpha_{1}+\alpha_{2}\right) \mathbf{m} \cdot \mathbf{x} .
\end{aligned}
$$

This expression has been written down by considering the sum of the real parts of terms (2.1) and (2.2) and multiplying by a similar sum. The coefficient vectors $\lambda$, $\mu$, et cetera, are independent of $\mathbf{x}$ and $t$. For the purpose in hand it is not necessary to know $\lambda$, $\mu$, et cetera, explicitly.

$\mathscr{E}$ will have a form similar to that of $\mathbf{R}$, the only difference being that the vectors $\lambda, \mu$ et cetera, will be replaced by scalars.

If $\mathbf{R}$ and $\mathscr{E}$ are now inserted into (1.3) and coefficients compared, it is found that

$$
\begin{aligned}
& \theta^{(1)} \cdot \mathbf{m}=0, \quad \theta^{(2)} \cdot \mathbf{m}=0, \\
& \left(\mathbf{S}^{(1)}-\mathbf{S}^{(2)}\right) \cdot \Psi^{+}=0, \\
& \left(\mathbf{S}^{(1)}-\mathbf{S}^{(2)}\right) \cdot \Psi^{-}=0 .
\end{aligned}
$$

There are other results but they are not germane to our purpose here.

In view of (2.3), the equations (2.5) may be written

$$
\theta^{(1)} \cdot m=\theta^{(2)} \cdot m=\Psi^{+} \cdot m=\Psi^{-} \cdot m=0 .
$$

If the mean energy flux vector is denoted by $\tilde{\mathbf{R}}$, then

$$
\tilde{\mathbf{R}}=(\omega / 2 \pi) \int_{0}^{2 \pi / \omega} \mathbf{R} d t
$$

From (2.4),

$$
\begin{aligned}
\tilde{\mathbf{R}}= & \boldsymbol{\theta}^{(1)} \exp -2 \alpha_{1} \mathbf{m} \cdot \mathbf{x}+\boldsymbol{\theta}^{(2)} \exp -2 \alpha_{2} \mathbf{m} \cdot \mathbf{x} \\
& +\left[\boldsymbol{\Psi}^{+} \cos \omega\left(\mathbf{S}^{(1)}-\mathbf{S}^{(2)}\right) \cdot \mathbf{x}\right. \\
& \left.-\boldsymbol{\Psi}^{-} \sin \omega\left(\mathbf{S}^{(1)}-\mathbf{S}^{(2)}\right) \cdot \mathbf{x}\right] \exp -\left(\alpha_{1}+\alpha_{2}\right) \mathbf{m} \cdot \mathbf{x} .
\end{aligned}
$$

The $\psi^{+}, \psi^{-}$terms represent the cross terms due to the superposition of the two fields.

† The real and imaginary parts of a complex quantity $K$ are denoted by $K^{+}$and $K^{-}$respectively. Thus $K=K^{+}+i K^{-}$. 
From (2.6) it now follows that

$$
\tilde{\mathbf{R}} \cdot \mathbf{m}=0 \text {. }
$$

Thus the mean energy flux vector for the combined fields is also parallel to the boundary. The result is valid for any number of surface waves since in forming $\mathbf{R}$ the individual fields combine in pairs.

\section{Reflection and refraction of linearly polarized waves}

Reflection and refraction of linearly polarized waves at a plane boundary is now considered.

Suppose an infinite train of linearly polarized waves is incident upon a plane boundary $\mathbf{m} . \mathbf{x}=\mathbf{0}$. It is first assumed that there is just one reflected wave train and that there are no refracted waves. It is also assumed that no energy is supplied at the boundary. Of course the two wave trains will interfere. The purpose here is to give a simple proof of the obvious physical fact that the component normal to the surface of the mean energy flux vector for the combined motion is equal to the sum of the components normal to the surface of the mean energy flux vectors of the two individual motions.

Let the incident field be represented by

$$
\mathbf{H} \exp i \omega(S \mathbf{n} . \mathbf{x}-t), \quad \mathbf{n} \cdot \mathbf{n}=1 \text {, }
$$

where $S$ and $\omega$ are real, and let the reflected field be represented by

$$
\mathrm{K} \exp i \omega(T \mathbf{p} \cdot \mathbf{x}-t), \mathbf{p} \cdot \mathbf{p}=1,
$$

where $T$ is real. The unit vectors $\mathbf{n}$ and $\mathbf{p}$ give the directions of the planes of constant phase.

The form of $\mathbf{R}$ for the combined field is

$$
\begin{aligned}
\mathbf{R}= & {\left[\alpha_{1}+\alpha_{2} \cos 2 \omega(S \mathbf{n} \cdot \mathbf{x}-t)+\alpha_{3} \sin 2 \omega(S \mathbf{n} \cdot \mathbf{x}-t)\right] } \\
& +\left[\beta_{1}+\beta_{2} \cos 2 \omega(T \mathbf{p} \cdot \mathbf{x}-t)+\beta_{3} \sin 2 \omega(T \mathbf{p} \cdot \mathbf{x}-t)\right] \\
& +\delta_{1} \cos \omega[(S \mathbf{n}+T \mathbf{p}) \cdot \mathbf{x}-2 t]+\delta_{2} \cos \omega\left[\left(S \mathbf{n}-T_{\mathbf{p}}\right) \cdot \mathbf{x}\right] \\
& +\delta_{3} \sin \omega[(S \mathbf{n}+T \mathbf{p}) \cdot \mathbf{x}-2 t]+\delta_{4} \sin \omega\left[\left(S \mathbf{n}-T_{\mathbf{p}}\right) \cdot \mathbf{x}\right],
\end{aligned}
$$

where $\alpha_{1}, \alpha_{2}, \alpha_{3}, \beta_{1}, \beta_{2}, \beta_{3}, \delta_{1}, \ldots, \ldots, \delta_{4}$, are real vectors independent of $x$ and $t$. The mean energy flux vector for the resultant motion is

$$
\tilde{\mathbf{R}}=\boldsymbol{\alpha}_{1}+\boldsymbol{\beta}_{1}+\boldsymbol{\delta}_{2} \cos \omega\left(\boldsymbol{S} \mathbf{n}-T_{\mathbf{p}}\right) \cdot \mathbf{x}+\boldsymbol{\delta}_{4} \sin \omega\left(S \mathbf{n}-T_{\mathbf{p}}\right) \cdot \mathbf{x} .
$$

The first term $\alpha_{1}$ may be identified with ${ }^{1} \tilde{\mathbf{R}}$ the mean energy flux vector associated with the incident wave train and similarly the mean energy flux vector associated with the reflected wave train is $\beta_{1}={ }^{2} \tilde{\mathbf{R}}$. The other terms $\delta_{2}$ and $\delta_{4}$ represent the interaction terms. Thus (3.4) may be written

$$
\tilde{\mathbf{R}}={ }^{1} \tilde{\mathbf{R}}+{ }^{2} \tilde{\mathbf{R}}+\boldsymbol{\delta}_{2} \cos \omega(S \mathbf{n}-T \mathbf{p}) \cdot \mathbf{x}+\boldsymbol{\delta}_{4} \sin \omega(S \mathbf{n}-T \mathbf{p}) \cdot \mathbf{x}
$$


The form of $\mathscr{E}$ is similar:

$$
\begin{aligned}
\mathscr{E}= & {\left[A_{1}+A_{2} \cos 2 \omega(S \mathbf{n} \cdot \mathbf{x}-t)+A_{3} \sin 2 \omega(S \mathbf{n} \cdot \mathbf{x}-t)\right] } \\
& +\left[B_{1}+B_{2} \cos 2 \omega(T \mathbf{p} \cdot \mathbf{x}-t)+B_{3} \sin 2 \omega(T \mathbf{p} \cdot \mathbf{x}-t)\right] \\
& +D_{1} \cos \omega\left[\left(S \mathbf{n}+T_{\mathbf{p}}\right) \cdot \mathbf{x}-2 t\right]+D_{2} \cos \omega\left[\left(S \mathbf{n}-T_{\mathbf{p}}\right) \cdot \mathbf{x}\right] \\
& +D_{3} \sin \omega\left[\left(S \mathbf{n}+T_{\mathbf{p}}\right) \cdot \mathbf{x}-2 t\right]+D_{4} \sin \omega\left[\left(S \mathbf{n}-T_{\mathbf{p}}\right) \cdot \mathbf{x}\right]+F,
\end{aligned}
$$

where $A_{1}, \ldots, A_{3}, B_{1}, \ldots, B_{3}, D_{1}, \ldots, D_{4}, E$ are real constants iñúpétendeñt ơ $\bar{x}$ and $\dot{t}_{\text {. }}$

Insert (3.3) and (3.6) into the conservation equation (1.3) to obtain

$$
(S \mathbf{n}-T \mathbf{p}) \cdot \delta_{2}=0, \quad(S \mathbf{n}-T \mathbf{p}) \cdot \delta_{4}=0,
$$

together with some other relations not relevant to the present work.

Now since the incident and reflected waves must be in phase on the boundary $\mathbf{m} . \mathbf{x}=\mathbf{0}$ it follows that

$$
(S \mathbf{n}-T \mathbf{p})=K \mathbf{m},
$$

where $K$ is some scalar. Thus by (3.7)

$$
\mathbf{m} \cdot \boldsymbol{\delta}_{2}=\mathbf{m} \cdot \boldsymbol{\delta}_{4}=0 \text {, }
$$

and from (3.5),

$$
\tilde{\mathbf{R}} \cdot \mathbf{m}={ }^{1} \tilde{\mathbf{R}} \cdot \mathbf{m}+{ }^{2} \tilde{\mathbf{R}} \cdot \mathbf{m} .
$$

This holds at every point of the region in which the waves propagate.

In forming $\mathbf{R}$ the fields combine in pairs and hence exactly the same argument will give a corresponding result for $q$ wave trains incident and reflected from a boundary:

$$
\tilde{\mathbf{R}} \cdot \mathbf{m}=\sum_{j=1}^{q}(j) \tilde{R} \cdot \mathbf{m} .
$$

Suppose now that $\mathbf{m} \cdot \mathbf{x}=0$ is the plane interface between two regions. If $\mathbf{R}_{(1)}$ and $\mathbf{R}_{(2)}$ are the energy flux vectors in the two regions, then applying an integral form of the energy conservation equation (1.3) to a "pill box" enclosing part of the boundary, and then shrinking the height of the pill box to zero, it follows in the usual way that

at the boundary.

$$
\left\{\mathbf{R}_{(\mathbf{1})}-\mathbf{R}_{(\mathbf{2})}\right\} \cdot \mathbf{m}=0
$$

Using equation (3.11) for each of the regions separately and equation (3.12), it follows that if there is any number of waves incident on the surface from either region with corresponding reflected and refracted waves, then in an obvious notation,

$$
\tilde{\mathbf{R}}_{(1)} \cdot \mathbf{m}=\sum_{j=1}^{q}{ }^{(j)} \tilde{\mathbf{R}}_{(1)} \cdot \mathbf{m}=\tilde{\mathbf{R}}_{(2)} \cdot \mathbf{m}=\sum_{k=1}^{\tau}{ }^{(k)} \tilde{\mathbf{R}}_{(2)} \cdot \mathbf{m},
$$


where there are $q$ waves in region " 1 " and $\tau$ waves in region " 2 ", and equation (3.13) is valid on the interface $\mathbf{m} \cdot \mathbf{x}=0$.

\section{Waves in unbounded regions}

In Sections 2 and 3 results were deduced for superposed wave trains in regions with boundaries. The purpose here is to consider the situation when there are no boundaries involved explicitly.

First consider the situation when a number of trains of linearly polarized waves all with the same frequency propagate in a direction $\mathbf{n}$. For example, in the case of an elastic crystal three wave trains may propagate in any direction [3]. Here it is assumed that there are $q$ wave trains propagating in the direction $\mathbf{n}$. Let the field be represented by

$$
\sum_{\alpha=1}^{q} \mathbf{A}_{(\alpha)} \operatorname{expi} \omega\left(S_{\alpha} \mathbf{n} \cdot \mathbf{x}-t\right), \quad \mathbf{n} \cdot \mathbf{n}=1 .
$$

Here $S_{\alpha}$ are assumed real and the $\mathbf{A}_{(\alpha)}$, which are scalars, vectors or tensors, are almost completely arbitrary. The qualification "almost completely" arises because there may be restrictions on the $\mathbf{A}_{(\alpha)}$ due to the physics of the situation. For example, in the case of an elastic crystal, if the three possible $\mathbf{A}_{(\alpha)}, \alpha=1,2,3$, are the three possible amplitude vectors, these must be mutually orthogonal [3].

The energy flux vector corresponding to (4.1) has the form

$$
\begin{aligned}
\mathbf{R}= & \beta_{1}+\sum_{\alpha=1}^{q} \beta_{2}^{(\alpha)} \cos 2 \omega\left(S_{\alpha} \mathbf{n} \cdot \mathbf{x}-t\right)+\sum_{\alpha=1}^{q} \beta_{3}^{(\alpha)} \sin 2 \omega\left(S_{\alpha} \mathbf{n} \cdot \mathbf{x}-t\right) \\
& +\sum_{\alpha=1}^{q} \sum_{\gamma=1}^{q}\left(\mu^{(\alpha, \gamma)}+\mu^{(\gamma, \alpha)}\right) \cos \omega\left[\left(S_{\alpha}+S_{\gamma}\right) \mathbf{n} \cdot \mathbf{x}-2 t\right] \\
& +\sum_{\alpha=1}^{q} \sum_{\gamma=1}^{q}\left(\theta^{(\alpha, \gamma)}+\theta^{(\gamma, \alpha)}\right) \cos \omega\left(S_{\alpha}-S_{\gamma}\right) \mathbf{n} \cdot \mathbf{x},
\end{aligned}
$$

where $\beta_{1}, \beta_{2}^{(\alpha)}, \beta_{3}^{(\alpha)}, \mu^{(\alpha, \gamma)} \theta^{(\alpha, \gamma)}$ are real vectors independent of $\mathbf{x}$ and $t$ and the asterisk denotes that $\alpha$ should not be equal to $\gamma$. Note that

$$
\tilde{\mathbf{R}}=\beta_{1}+{ }^{*} \sum_{\alpha=1}^{q} \sum_{\gamma=1}^{q}\left(\theta^{(\alpha, \gamma)}+\theta^{(\gamma, \alpha)}\right) \cos \omega\left(S_{\alpha}-S_{\gamma}\right) \mathbf{n} \cdot \mathbf{x} .
$$

The first term, $\beta_{1}$, is the vector sum of the mean energy flux vectors ${ }^{(\alpha)} \tilde{\mathbf{R}}$, $\alpha=1, \ldots, q$, of the individual motions, and the second term in (4.3) represents the interaction terms.

$\mathscr{E}$ will have a form similar to (4.2). Inserting $\mathbf{R}$ and $\mathscr{E}$ in (1.3) leads to

$$
\left(\theta^{(\alpha, \gamma)}+\theta^{(\gamma, \alpha)}\right) \cdot \mathbf{n}=0, \quad \alpha, \gamma=1,2, \ldots, q, \quad \alpha \neq \gamma .
$$


Thus

$$
\tilde{\mathbf{R}} \cdot \mathbf{n}=\boldsymbol{\beta}_{\mathbf{1}} \cdot \mathbf{n}=\sum_{\alpha=1}^{q}(\alpha) \tilde{\mathbf{R}} \cdot \mathbf{n} .
$$

In other words: the component in the direction of $\mathbf{n}$ of the mean energy flux of the resultant motion is equal to the algebraic sum of the components in the direction of $\mathbf{n}$ of the mean energy flux vectors for the individual motions.

Now consider two wave trains propagating in arbitrary directions. In Section 3 for the two trains (3.1) and (3.2), it was seen that

$$
\widetilde{\mathbf{R}}={ }^{1} \tilde{\mathbf{R}}+{ }^{2} \widetilde{\mathbf{R}}+\delta_{2} \cos \omega(S \mathbf{n}-T \mathbf{p}) \cdot \mathbf{x}+\boldsymbol{\delta}_{4} \sin \omega\left(S \mathbf{n}-T_{\mathbf{p}}\right) \cdot \mathbf{x},
$$

and from the energy equation it was shown that $(S n-T p) \cdot \delta_{2}=0,(S n-T p) \cdot \delta_{4}=0$. Hence

$$
\tilde{\mathbf{R}} \cdot\left(\mathbf{S}_{(1)}-\mathbf{S}_{(2)}\right)=\left({ }^{2} \tilde{\mathbf{R}}+{ }^{2} \tilde{\mathbf{R}}\right) \cdot\left(\mathbf{S}_{(1)}-\mathbf{S}_{(2)}\right),
$$

where I have written $\mathbf{S}_{(1)}=S \mathbf{n}, \mathbf{S}_{(2)}=T \mathbf{p} . \mathbf{S}_{(1)}$ and $\mathbf{S}_{(2)}$ are then two slownesses [3].

I think that (4.7) is probably the best that can be achieved in general. For the mean energy flux vector for two wave trains need not even lie in the plane spanned by the two mean energy flux vectors for the individual wave trains: An example from the classical linear theory of homogeneous isotropic elastic bodies will illustrate this.

Let the shear modulus of the material be denoted by $\mu$ and the density by $\rho$. The displacement vector $u_{1}$ corresponding to a transverse wave propagating in the direction $\mathbf{m}$ may be written

$$
\mathbf{u}_{1}=\mathbf{A} \cos \omega(S \mathbf{m} \cdot \mathbf{x}-t), \quad \mathbf{A} \cdot \mathbf{m}=0 .
$$

This wave may propagate provided the slowness $S$ is given by

$$
\mu S^{2}=\rho .
$$

$\mathbf{A}$ is any vector at right angles to $\mathbf{m}$. The corresponding stress $t_{i j}$ is given by

$$
t_{i j}=-\mu\left(A_{i} m_{j}+A_{j} m_{i}\right) \omega S \sin \omega(S \mathrm{~m} \cdot \mathbf{x}-t),
$$

and the mean energy flux vector is

$$
{ }^{1} \tilde{\mathbf{R}}=\left[\mu \omega^{2} S(\mathbf{A} . \mathbf{A}) / 2\right] \mathbf{m} .
$$

Similarly, for the transverse wave

$$
\mathbf{u}_{2}=\mathbf{B} \cos \omega(S \mathbf{p} \cdot \mathbf{x}-t), \quad \beta \cdot \mathbf{p}=0,
$$

with $S$ given by (4.9), and $\mathbf{B}$ any vector at right angles to $\mathbf{p}$, the mean energy flux vector is

$$
{ }^{2} \tilde{\mathbf{R}}=\left[\mu \omega^{2} s \mathbf{B} \cdot \mathbf{B} / 2\right] \mathbf{p} .
$$


However, for the combined motion $u_{1}+u_{2}$ the energy flux vector is

$$
\begin{aligned}
\mathbf{R}= & \mu \omega^{2} S\left\{\left[(\mathbf{A} \cdot \mathbf{A}) \sin ^{2} \omega(S \mathbf{m} \cdot \mathbf{x}-t)\right] \mathbf{m}+\left[(\mathbf{B} \cdot \mathbf{B}) \sin ^{2} \omega(S \mathbf{p} \cdot \mathbf{x}-t)\right] \mathbf{p}\right. \\
& +[(\mathbf{A} \cdot \mathbf{B})(\mathbf{p}+\mathbf{m})+(\mathbf{A} \cdot \mathbf{p}) \mathbf{B}+(\mathbf{B} \cdot \mathbf{m}) \mathbf{A}] \times \sin \omega(S \mathbf{m} \cdot \mathbf{x}-t) \\
& \times \sin \omega(S \mathbf{p} \cdot \mathbf{x}-t)\} .
\end{aligned}
$$

Thus the mean energy flux for the combined motion is given by

$$
\begin{aligned}
2 \tilde{\mathbf{R}}=2^{1} \tilde{\mathbf{R}}+2^{2} \tilde{\mathbf{R}} & +[(\mathbf{A} \cdot \mathbf{B})(\mathbf{p}+\mathbf{m})+(\mathbf{A} \cdot \mathbf{p}) \mathbf{B} \\
& +(\mathbf{B} \cdot \mathbf{m}) \mathbf{A}] \cos \omega S(\mathbf{m}-\mathbf{p}) \cdot \mathbf{x} .
\end{aligned}
$$

Choose, for example, $m=\mathbf{i}, \mathbf{p}=\mathbf{j}, \mathbf{A}=\mathbf{j}$ and $\mathbf{B}=\mathbf{k}$. Then

$$
2\left(\tilde{\mathbf{R}}-{ }^{1} \tilde{\mathbf{R}}-{ }^{2} \tilde{\mathbf{R}}\right)=\mathbf{k} \cos \omega S(\mathbf{i}-\mathbf{j}) \cdot \mathbf{x} \text {. }
$$

It is seen that $\tilde{\mathbf{R}} \cdot \mathbf{k} \neq 0$ even though ${ }^{1} \tilde{\mathbf{R}} \cdot \mathbf{k}=0$ and ${ }^{2} \tilde{\mathbf{R}} \cdot \mathbf{k}=0$.

From (4.15)

$$
\tilde{\mathbf{R}} \cdot(\mathbf{p}-\mathbf{m})=\left({ }^{1} \tilde{\mathbf{R}}+{ }^{2} \tilde{\mathbf{R}}\right) \cdot(\mathbf{p}-\mathbf{m}),
$$

confirming equation (4.7) in this case.

\section{REFERENCES}

[1] M. Hayes, "A note on plane waves in linear conservative systems", Q.Jl. Mech. Appl. Math. 28 (1975), 329-332.

[2] D. S. Jones, The Theory of Electromagnetism, Pergamon Press, London (1964).

[3] M. J. P. Musgrave, Crystal Acoustics, Holden Day, San Francisco (1970).

[4] J. A. Schouten, Tensor Analysis for Physicists, 2nd ed., Clarendon Press, Oxford (1959).

[5] J. L. Synge, "Flux of energy for elastic waves in anisotropic media", Proc. R. Ir. Acad. 58A (1956), 13-20.

Department of Mathematical physics

University College

Belfield

Dublin, Ireland 